\title{
QUEUING SYSTEM IN SYNCHRONOUS OPTICAL NETWORK (SONET)
}

S. Maragathasundari

Associate professor, Department of Mathematics, Kalasalingam Academy of Research and Education.

Krishnankoil, (India). E-mail: maragatham01@gmail.com ORCID: https://orcid.org/0000-0003-1210-6411

\section{P. Suthersan}

Assistant professor, Department of Mathematics, Kalasalingam Academy of Research and Education. Krishnankoil, (India). E-mail: suthersan.p@klu.ac.in ORCID: http://orcid.org/0000-0003-0712-0518

K. S. Dhanalakshmi

Assistant professor, Department of Electronics and Communication Engineering, Kalasalingam Academy of Research and Education.

Krishnankoil, (India).

E-mail: k.s.dhanalakshmi@klu.ac.in ORCID: https://orcid.org/0000-0001-6285-3656

\section{Citación sugerida:}

Maragathasundari, S., Suthersan, P., y Dhanalakshmi, K. S. (2020). Queuing system in Synchronous Optical Network (SONET). 3C Tecnología. Glosas de innovación aplicadas a la pyme. Edición Especial, Marzo 2020, 231-245. http://doi.org/10.17993/3ctecno.2020.specialissue4.231-245

\section{Suggested citation:}

Maragathasundari, S., Suthersan, P., \& Dhanalakshmi, K. S. (2020). Queuing system in Synchronous Optical Network (SONET). 3C Tecnología. Glosas de innovación aplicadas a la pyme. Edición Especial, Marzo 2020, 231-245. http://doi.org/10.17993/3ctecno.2020.specialissue4.231-245 


\section{ABSTRACT}

This paper researches an investigation on Queuing framework in Synchronous Optical Network (SONET). Optical fiber utilized in SONET can blame on various conditions that are capricious, which is a fundamental dependability worry for power lattice interchanges. Different transmission advances have been utilized in whole deal interchanges, for example, optical fiber, microwave, or satellite. Optical fiber can blame on various flighty conditions which make it a noteworthy danger to arrange unwavering quality. Phases of administration in SONET, administration intrusion in this system are well explained. The Queuing issue occurring in this system is very much tackled by strengthening variable methodology and the comparing line execution measures are inferred. The purpose of issue emerged is very much anticipated by this Queuing approach and the administration interference could be limited or to a NIL base. Numerical delineation encourages the model to be defended to an incredible extent. Graphical portrayal unmistakably clarifies the presentation proportions of the Queuing framework in SONET.

\section{KEYWORDS}

Batch arrival, Optional First Stage, Compulsory Second Stage, Service Interruption. 


\section{INTRODUCTION}

SONET is utilized to change over electrical sign into optical sign so it can travel longer separations. Synchronous Optical NET work (SONET) is a typical for optical televise communications transport, which was created in the mid-1980s, and stays in boundless use today. Contrasted with Ethernet cabling that traverses separations up to100 meters, SONET fiber ordinarily runs a lot further. Indeed, even short achieve connections range as much as 2 kilometers (1.2 miles); intermediate and long achieve connections spread many kilometers. Along these lines it is appropriate for whole deal transmission, for example, the one in the power lattice correspondences. Wu, Kobrinski, Ghosal, and Lakshman (1994) examined a few DCS design upgrade choices, including a parallel handling/crossinterface DCS engineering, which may improve the administration rebuilding time. Boehm, Ching, Griffith, and Saal (1986) gave an account of the exercises in different benchmarks associations, with accentuation on a synchronous system proposition which is as of now being talked about in the $\mathrm{T} 1$ advisory group. Way, Smith, Johnson, and Izadpanah (1992) tentatively confirmed the system idea and talked about various system applications for bursty information traffic and persistent voice/video traffic. Blumenthal et al. (2003) explored the sign handling procedures, Hac and Mutlu (1989) researched the B-ISDN convention, guidelines utilized in the Broadband reference model. Lee, Sherali, Han, and Kim (2000) dealt with a system plan issue emerging from the sending of synchronous optical systems (SONET), a standard of transmission utilizing optical fiber innovation. Cosares, Deutsch, Saniee, and Wasem (1995) inspected SONET framework by the Bellcore customer organizations has spared 10 to 30 percent in expenses and requests of greatness in time. Chao, Shtirmer, and Smoot (1989) broke down the physical layer of the system utilizes the synchronous optical system transmission design. Fundamental ideas are talked about and reviewed by Jue, Yang, Kim, and Zhang (2009). Kang, Park, Shin, and Jeong (1995) watched the normal for the system relying on the collected transmission limit of the network. Maragathasundari and Balamurugan (2015) contemplated the presentation examination of bunch landing line with two phases of administration. Maragathasundari and Dhanalakshmi (2018) investigated versatile adhoc systems issue A Queuing approach. Maragathasundari and Srinivasan (2012) made an investigation on M/G/1 input line with three phase and different server get-away. Maragathasundari and Srinivasan (2015) examined a Non-Markovian Multistage Batch entry line with breakdown and reneging. An examination on the investigation of execution proportion of mass information line with $\mathrm{N}$ 
sort of extra discretionary administration, administration interference and deterministic get-away were inspected by Maragathasundari and Sowmiah (2016).

\subsection{ADVANTAGES OF SONET}

1) Transmits data to large distances.

2) Low electromagnetic interference.

3) High data rates.

4) Large Bandwidth.

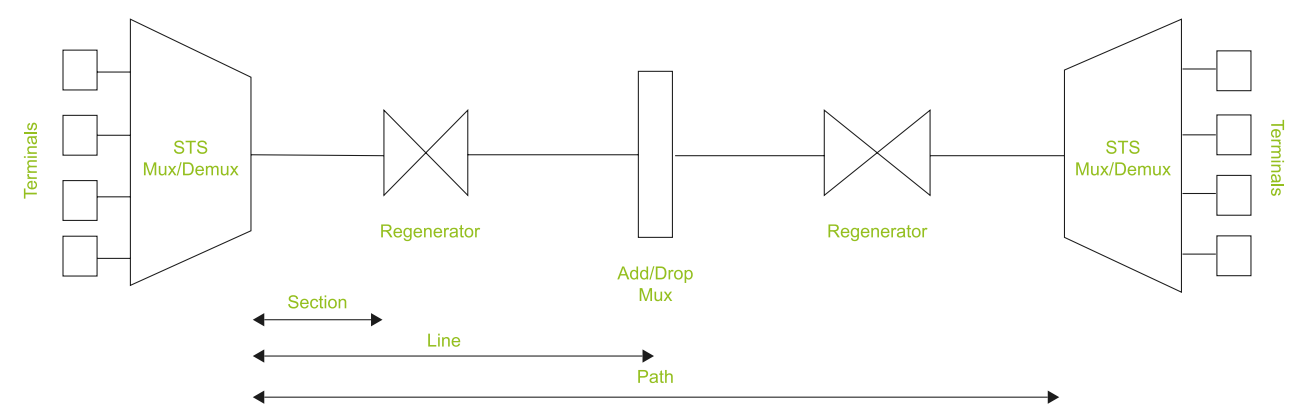

Graphic 1. SONET Network Elements.

\subsection{SONET CONNECTIONS}

1) Section: Portion of system interfacing two neighboring gadgets.

2) Line: Portion of system interfacing two neighboring multiplexers.

3) Path: End-to-end segment of the system.

\section{*STS Multiplexer:}

Performs multiplexing of sign.

\section{*STS Demultiplexer:}

Performs demultiplexing of sign.

Converts optical sign to electrical sign.

\section{*Regenerator:}

It is a repeater, that takes an optical sign and recovers (builds the quality) it. 


\section{*Add/Drop Multiplexer:}

It permits including sign originating from various sources into a given way or expelling a sign.

\subsection{SONET LAYERS}
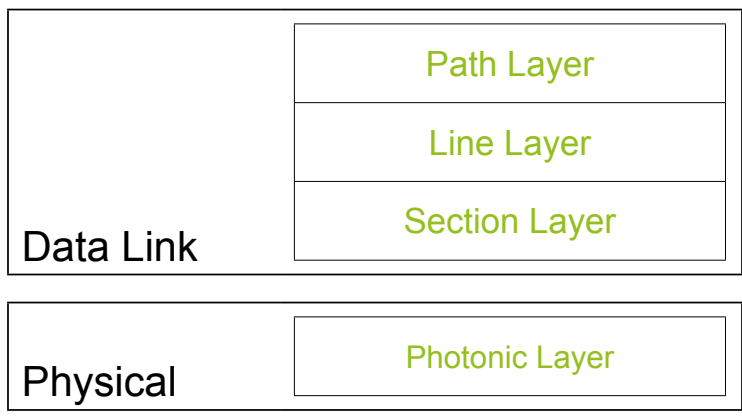

Graphic 2. SONET Layers.

SONET includes four functional layers:

\section{1) Path Layer:}

a. It is in charge of the development of sign from its optical source to its optical goal.

b. STS Mux/Demux gives way layer capacities.

\section{2) Line Layer:}

a. It is in charge of the development of sign over a physical line.

b. STS Mux/Demux and Add/Drop Mux give Line layer capacities.

\section{3) Section Layer:}

a. It is in charge of the development of sign over a physical area.

b. Each gadget of system gives segment layer capacities.

\section{4) Photonic Layer:}

a. It relates to the physical layer of the OSI model.

b. It incorporates physical determinations for the optical fiber channel (nearness of light $=1$ and nonappearance of light $=0$ ). 


\subsection{PERFORMANCE REQUIREMENTS}

We assume the following to describe the queuing model of our study.

1) Batch arrival - Queue - We consider a solitary server line which will give two distinct administrations, an Essential Service and Optional Service.

2) Essential administration - 2 phases: One of the benefits of SONET is that it can pass on gigantic payloads (more than $50 \mathrm{Mbps}$ ). To achieve this capacity, the STS SPE can be sub-apportioned into more diminutive sections or structures, known as VTs (Virtual tributaries)

*Optional First Stage: Except for connected sign, all data sources are at last changed over to a base setup of a synchronous STS-1 signal (51.84 Mbps or higher). Lowerspeed information sources, for instance, DS-1s are first piece or byte-multiplexed into VTs. Several synchronous STS-1s are then multiplexed together in either a singular or two-mastermind system to outline an electrical STS $-\mathrm{N}$ signal $(\mathrm{N}>=1)$.

* Compulsory Second Stage: Any kind of organization, running from voice to quick data and video, can be recognized by various types of organization connectors. An organization connector maps the sign into the payload envelope of the STS-1 or VT. New organizations and sign can be transported by including new organization connectors at the edge of the SONET sort out.

3) Optional administration - Service Interruption happens - Optical Cable Failures are considered here as Service Interruption during this Optional Service.

Three kinds of optical strands have been utilized in the whole deal transport of information.

- Buried fiber optic links have a higher disappointment rate than the two overhead links.

- Optical ground wire links and introduced overhead on posts or transmission towers.

- All dielectric self-supporting cables introduced overhead on posts or transmission towers. 
Two sorts of disappointments are being considered,

(a) "cable cut" disappointments which will influence both the working strand and the assurance strand, and

(b) "Strand disappointments" which will bomb just one strand inside the link.

4) Completion of Both administrations- Dissatisfied Customers (not ready to utilize the multiplexing procedure successfully) can join the tail of the first line to get a Feedback administration.

\section{MATHEMATICAL PORTRAYAL OF THE QUEUING MODEL}

The arithmetical portrayal of the Queuing frame work has the option to be described by the resulting proposition:

Customers meet up at the structure in clusters of variable size in a compound strategy pursues Poisson conveyance. Let $\lambda d_{j} d t(j=1,2,3 \ldots)$ be the first order probability that a batch of $j$ customers arrives at the system during a short duration of time $(t, t+d t)$ where $0 \leq d_{j} \leq 1$ and $\sum_{j=1}^{n} d_{j}=1$ and $\lambda>0$ is the mean landing rate of the batches.

The administration time pursues general(arbitrary) circulation. First stage of essential service follows distribution function as $L_{e_{1}}(x)$ and density function $l_{e_{1}}(x)$. Let $\mu_{\left(e_{1}\right)}(x) d x$ be the conditional density function. Hence, we have:

$$
\mu_{\left(e_{1}\right)}(x)=\frac{l_{e_{1}}(x)}{1-L_{e_{1}}(x)}, \quad l_{e_{1}}(x)=\mu_{\left(e_{1}\right)}(x) e^{-\int_{0}^{s} \mu_{\left(e_{1}\right)}(s) d s}
$$

For second stage of essential service,

$$
\mu_{\left(e_{2}\right)}(x)=\frac{l_{e_{2}}(x)}{1-L_{e_{2}}(x)} \quad, l_{e_{2}}(x)=\mu_{\left(e_{2}\right)}(x) e^{-\int_{0}^{s} \mu_{\left(e_{2}\right)}(s) d s}
$$

For optional service,

$$
\mu_{k}(x)=\frac{l_{k}(x)}{1-L_{k}(x)} \quad, l_{k}(x)=\mu_{k}(x) e^{-\int_{0}^{s} \mu_{k}(s) d s}
$$


Service interruption follows Poisson distribution with mean rate $\beta>0$.

\section{GOVERNING EQUATIONS OF THE MODEL}

$$
\begin{gathered}
\frac{d}{d x} P_{n}^{\left(e_{1}\right)}(x)+\left(\lambda+\mu_{\left(e_{1}\right)}(x)\right) P_{n}^{\left(e_{1}\right)}(x)=\lambda \sum_{j=1}^{n} d_{j} P_{n-j}^{\left(e_{1}\right)}(x) \\
\frac{d}{d x} P_{0}^{\left(e_{1}\right)}(x)+\left(\lambda+\mu_{\left(e_{1}\right)}(x)\right) P_{0}^{\left(e_{1}\right)}(x)=0 \\
\frac{d}{d x} P_{n}^{\left(e_{2}\right)}(x)+\left(\lambda+\mu_{\left(e_{2}\right)}(x)\right) P_{n}^{\left(e_{2}\right)}(x)=\lambda \sum_{j=1}^{n} d_{j} P_{n-j}^{\left(e_{2}\right)}(x) \\
\frac{d}{d x} P_{0}^{\left(e_{2}\right)}(x)+\left(\lambda+\mu_{\left(e_{2}\right)}(x)\right) P_{0}^{\left(e_{2}\right)}(x)=0 \\
\frac{d}{d x} K_{n}(x)+\left(\lambda+\mu_{k}(x)+\beta\right) K_{n}(x)=\lambda \sum_{j=1}^{n} d_{j} K_{n-j}(x) \\
\frac{d}{d x} K_{0}(x)+\left(\lambda+\mu_{k}(x)+\beta\right) K_{0}(x)=0 \\
\frac{d}{d x} M_{n}(x)+\left(\lambda+\mu_{m}(x)\right) M_{n}(x)=\lambda \sum_{j=1}^{n} d_{j} M_{n-j}(x) \\
\frac{d}{d x} M_{0}(x)+\left(\lambda+\mu_{m}(x)\right) M_{n}(x)=0 \\
\lambda Q=\int_{0}^{\infty} M_{0}(x) \mu_{m}(x) d x+(1-m) \int_{0}^{\infty} P_{0}^{\left(e_{2}\right)}(x) \mu_{\left(e_{2}\right)}(x) d x+(1-r) Q \int_{0}^{\infty} K_{0}(x) \mu_{k}(x)
\end{gathered}
$$

\section{BOUNDARY CONDITIONS}

The following boundary conditions are used to solve the above equations:

$$
\begin{gathered}
P_{n}^{\left(e_{1}\right)}(0)=\int_{0}^{\infty} M_{n+1}(x) \mu_{m}(x) d x+(1-m) \int_{0}^{\infty} P_{n+1}^{\left(e_{2}\right)}(x) \mu_{\left(e_{2}\right)}(x) d x+r \int_{0}^{\infty} K_{n}(x) \mu_{k}(x) d x+\lambda D_{n+1} \mathrm{Q} \\
P_{n}^{\left(e_{2}\right)}(0)=\int_{0}^{\infty} P_{n}^{\left(e_{1}\right)}(x) \mu_{\left(e_{1}\right)}(x) d x \\
K_{n}(0)=\int_{0}^{\infty} P_{n}^{\left(e_{2}\right)}(x) \mu_{\left(e_{2}\right)} d x \\
M_{n}(0)=\beta \int_{0}^{\infty} K_{n}(x)
\end{gathered}
$$




\section{QUEUE LENGTH DISTRIBUTION}

\section{Usage of Supplementary variable technique}

We multiply (1) by $z^{n}$ and sum over $\mathrm{n}$ from 1 to $\infty$ and add it to (2).

We get,

$$
\frac{d}{d x} P^{\left(e_{1}\right)}(x, z)+\left(\lambda-\lambda D(z)+\mu_{\left(e_{1}\right)}(x)\right) P^{\left(e_{1}\right)}(x, z)=0
$$

Again integrating the above from 0 to $\mathrm{n}$, we get

$$
P^{\left(e_{1}\right)}(x, z)=P^{\left(e_{1}\right)}(0, z) e^{-(\lambda-\lambda D(z)) x} \int_{0}^{x} \mu_{\left(e_{1}\right)}(t) d t
$$

Again integrating $\left(^{*}\right)$ by parts with respect to $x$ yields,

$$
P^{\left(e_{1}\right)}(z)=P^{\left(e_{1}\right)}(0, z)\left[\frac{1-L_{\left(e_{1}\right)}(\lambda-\lambda D(z))}{(\lambda-\lambda D(z))}\right]
$$

Multiplying both sides of the $\left.{ }^{*}\right)$ by $\mu_{\left(e_{1}\right)}(x)$ and integrating over $x$, we get:

$$
\int_{0}^{\infty} P^{\left(e_{1}\right)}(x, z) \mu_{\left(e_{1}\right)}(x)=P^{\left(e_{1}\right)}(0, z) L_{\left(e_{1}\right)}(\lambda-\lambda D(z))
$$

Applying the same concept for the second stage (optional) in essential service $\left[P_{n}^{\left(e_{1}\right)}(x)\right]$, optional service $\left[K_{n}(x)\right]$, and repair process $\left[M_{n}(x)\right]$, we get,

$$
\begin{gathered}
\text { i) } P^{\left(e_{2}\right)}(z)=P^{\left(e_{2}\right)}(0, z)\left[\frac{1-L_{\left(e_{2}\right)}(\lambda-\lambda D(z))}{(\lambda-\lambda D(z))}\right] \\
=P^{\left(e_{1}\right)}(0, z) L_{\left(e_{1}\right)}(\lambda-\lambda D(z))\left[\frac{1-L_{\left(e_{2}\right)}(\lambda-\lambda D(z))}{(\lambda-\lambda D(z))}\right]
\end{gathered}
$$

Also we have,

$$
\begin{array}{r}
\int_{0}^{\infty} P^{\left(e_{2}\right)}(x, z) \mu_{\left(e_{2}\right)}(x) d x=P^{\left(e_{1}\right)}(0, z) L_{\left(e_{1}\right)}(\lambda-\lambda D(z)) L_{\left(e_{2}\right)}(\lambda-\lambda D(z)) \\
\text { ii) } K(z)=K(0, z)\left[\frac{1-L_{k}(\lambda-\lambda D(z)+\beta)}{(\lambda-\lambda D(z)+\beta)}\right]=P^{\left(e_{1}\right)}(0, z) L_{\left(e_{1}\right)}(\lambda-\lambda D(z))
\end{array}
$$




$$
L_{\left(e_{2}\right)}(\lambda-\lambda D(z))\left[\frac{1-L_{\left(e_{2}\right)}(\lambda-\lambda D(z))}{(\lambda-\lambda D(z))}\right]
$$

$\int_{0}^{\infty} K(x, z) \mu_{k}(x) d x=P^{\left(e_{1}\right)}(0, z) L_{\left(e_{1}\right)}(\lambda-\lambda D(z)) L_{\left(e_{2}\right)}(\lambda-\lambda D(z)) L_{k}(\lambda-\lambda D(z)+\beta)$

$M(z)=\beta z P^{\left(e_{1}\right)}(0, z) L_{\left(e_{1}\right)}(\lambda-\lambda D(z)) L_{\left(e_{2}\right)}(\lambda-\lambda D(z))\left[\frac{1-L_{k}(\lambda-\lambda D(z)+\beta)}{(\lambda-\lambda D(z)+\beta)}\right]\left[\frac{1-H(\lambda-\lambda D(z))}{(\lambda-\lambda D(z))}\right]$

$\int_{0}^{\infty} M(x, z) \mu_{m}(x) d x=\beta z^{\left(e_{1}\right)}(0, z) L_{\left(e_{1}\right)}(\lambda-\lambda D(z)) L_{\left(e_{2}\right)}(\lambda-\lambda D(z))\left[\frac{1-L_{k}(\lambda-\lambda D(z)+\beta)}{(\lambda-\lambda D(z)+\beta)}\right] H(\lambda-\lambda D(z))$

Now $\sum_{n=0}^{\infty} z^{n}(10)$, using (9) and further using (18), (20), (22) we get,

$$
P^{\left(e_{1}\right)}(0, z)=\frac{\lambda Q(D(z)-1)}{z-L_{\left(e_{1}\right)}(a) L_{\left(e_{2}\right)}(a)\left[\beta z H(a)\left[\frac{1-L_{k}(b)}{b}\right]+(1-m)+r L_{k}(b)\right]}
$$

Substituting (23) in (15), (17), (19), (21) we get,

$$
\begin{aligned}
& P^{\left(e_{1}\right)}(z)=\frac{\left[L_{\left(e_{1}\right)}(a)-1\right] Q}{z-L_{\left(e_{1}\right)}(a) L_{\left(e_{2}\right)}(a)\left[\beta z H(a)\left[\frac{1-L_{k}(b)}{b}\right]+(1-m)+r L_{k}(b)\right]} \\
& P^{\left(e_{2}\right)}(z)=\frac{L_{\left(e_{1}\right)}(a)\left[L_{\left(e_{2}\right)}(a)-1\right] Q}{z-L_{\left(e_{1}\right)}(a) L_{\left(e_{2}\right)}(a)\left[\beta z H(a)\left[\frac{1-L_{k}(b)}{b}\right]+(1-m)+r L_{k}(b)\right]} \\
& K(z)=\frac{a L_{\left(e_{1}\right)}(a) L_{\left(e_{2}\right)}(a)\left[L_{k}(b)-1\right]}{b\left[z^{\left.\left.\left.-L_{(}\right)^{(a) L_{(}}\right)^{(a)}\left[\beta z H(a)\left[\frac{-L_{k}(b)}{b}\right]+(1-m)+r L_{k}(b)\right]\right]}\right.} \\
& M(z)=\frac{\beta z L_{\left(e_{1}\right)}(a) L_{\left(e_{2}\right)}(a)\left[L_{k}(b)-1\right][1-H(a)]}{b\left[z-L_{\left(e_{1}\right)}(a) L_{\left(e_{2}\right)}(a)\left[\beta z H(a)\left[\frac{1-L_{k}(b)}{b}\right]+(1-m)+r L_{k}(b)\right]\right]}
\end{aligned}
$$

Where, $a=\lambda-\lambda D(z), b=\lambda-\lambda D(z)+\beta$

\section{PROBABILITY CAPACITY FUNCTION OF THE QUEUE LENGTH}

Let $J_{q}(z)$ be the PGFof the queue length

$$
J_{q}(z)=P^{\left(e_{1}\right)}(z)+P^{\left(e_{2}\right)}(z)+K(z)+M(z)
$$

Adding (24) to (27), we get, 


$$
J_{q}(z)=\frac{Q\left\{b\left[L_{\left(e_{1}\right)}(a) L_{\left(e_{2}\right)}(a)-1\right]+L_{\left(e_{1}\right)}(a) L_{\left(e_{2}\right)}(a)\left[L_{k}(b)-1\right][a+\beta z[1-H(a)]]\right\}}{b\left[z-L_{\left(e_{1}\right)}(a) L_{\left(e_{2}\right)}(a)\left[\beta z H(a)\left[\frac{1-L_{k}(b)}{b}\right]+(1-m)+r L_{k}(b)\right]\right]}
$$

\section{IDLE TIME AND UTILIZATION FACTOR}

Idle time is determined using the condition:

$$
J_{q}(1)+Q=1
$$

Applying LH rule we get,

$$
\begin{aligned}
& J_{q}(z)=\frac{N^{\prime}(1)}{D^{\prime}(1)} \\
& Q=\frac{D^{\prime}(1)}{D^{\prime}(1)+N^{\prime}(1)}
\end{aligned}
$$

From the idle factor $Q$, the utilization rate is calculated.

To find $L_{q}$ the length of the Queue and the Queue performance measures.

We have $L_{q}(z)=\frac{d}{d z} J_{q}(z) \mid z=1=\frac{0}{0}$ (indeterminate form)

$$
L_{q}=\lim _{z \rightarrow 1} \frac{D^{\prime}(z) N^{\prime \prime}(z)-N^{\prime}(z) D^{\prime \prime}(z)}{2\left(D^{\prime}(z)\right)^{2}}
$$

Here $J_{q}(z)=\frac{N(z)}{D(z)}$ where $\mathrm{N}(\mathrm{z})$ and $\mathrm{D}(\mathrm{z})$ are the numerator and denominator of (28).

$$
\begin{gathered}
N^{\prime}(1)=\lambda E(I)\left[E\left(L_{\left(e_{1}\right)}\right)+E\left(L_{\left(\left(_{2}\right)\right.}\right)+1+\beta E(H)\left(L_{k}(\beta)-1\right)\right] \\
N^{\prime \prime}(1)=2(-\lambda E(I))^{2}\left[E\left(L_{\left(e_{1}\right)}\right)+E\left(L_{\left(e_{2}\right)}\right)\right] \\
+\beta(\lambda E(I))^{2}\left[E\left(L_{\left(e_{1}\right)}{ }^{2}\right)+E\left(L_{\left(e_{2}\right)}{ }^{2}\right)+2\left[E\left(L_{\left(e_{1}\right)}\right) E\left(L_{\left(\left(_{2}\right)\right.}\right)\right]\right](-\lambda E(I))^{2}\left[-E(H)\left[E\left(L_{\left(\left(_{1}\right)\right.}\right)+E\left(L_{\left(\left(_{2}\right)\right.}\right)+L_{k}^{\prime}-\beta\right]\right. \\
\left.+\beta E(H)\left(L_{k}(\beta)+(-\lambda E(I))\left(E\left(L_{\left(e_{1}\right)}\right)+E\left(L_{\left(\left(_{2}\right)\right.}\right)+L_{k^{\prime}}\right)\right)+(-\lambda E(I))^{2} \beta E\left(H^{2}\right) L_{k}(\beta)\right] \\
D^{\prime}(1)=(-\lambda E(I))\left[1-\left(1-L_{k}(\beta)+(1-m)+r L_{k}(\beta)\right)\right] \\
+\beta\left\{1+(-\lambda E(I))\left[E\left(L_{\left(e_{1}\right)}\right)+E\left(L_{\left(\left(_{2}\right)\right.}\right)\right]\left[\left(1-L_{k}(\beta)\right)+(1-m)+r L_{k}(\beta)\right]\right. \\
\left.-\left[\left(1-L_{k}(\beta)\right)+\lambda E(I) E(H)\left(1-L_{k}(\beta)\right)+\beta \lambda E(I) L_{k}^{\prime}+r L_{k}^{\prime} \lambda E(I)\right]\right\}
\end{gathered}
$$




$$
\begin{aligned}
D^{\prime \prime}(1)= & -\lambda E(1) 2\left\{1+(-\lambda E(I))\left[E\left(L_{\left(e_{1}\right)}\right)+E\left(L_{\left(e_{2}\right)}\right)\right]\left[\left(1-L_{k}(\beta)\right)+(1-m)+r L_{k}(\beta)\right]\right. \\
- & {\left.\left[\left(1-L_{k}(\beta)\right]+\lambda E(I) E(H)\left(1-L_{k}(\beta)\right)+\beta \lambda E(I) L^{\prime}{ }_{k}+r L_{k}{ }_{k} \lambda E(I)\right]\right\} } \\
+ & \beta\left\{-(-\lambda E(I))^{2}\left(E\left(L_{\left(e_{1}\right)}{ }^{2}\right)+E\left(L_{\left(e_{2}\right)}{ }^{2}\right)+2 E\left(L_{\left(e_{1}\right)}\right) E\left(L_{\left(e_{2}\right)}\right)\right)\left[1-L_{k}(\beta)\right.\right. \\
& \left.+(1-m)+r L_{k}(\beta)\right]+(-\lambda E(I)) L^{\prime}{ }_{k}\left(E\left(L_{\left(e_{1}\right)}\right)+E\left(L_{\left(e_{2}\right)}\right)\right) \\
& -(\lambda E(1))\left(E ( L _ { ( e _ { 1 } ) } + E ( L _ { ( e _ { 2 } ) } ) ) \left[1-L_{k}(\beta)+E(H) \lambda E(I)\left(1-L_{k}(\beta)\right)\right.\right. \\
& \left.-\lambda E(I) L^{\prime}{ }_{k}+r \lambda E(I) L_{k}{ }_{k}\right] \\
& -\left[(\lambda E(I)) E(H)\left(1-L_{k}(\beta)\right)+\beta L^{\prime}{ }_{k}+E(H) \lambda E(I)\left(1-L_{k}(\beta)\right)\right. \\
+ & E\left(H^{2}\right)(-\lambda E(I))^{2}\left(1-L^{\prime}{ }_{k}(\beta)\right)+\beta E(I) E(H) L^{\prime}{ }_{k}+\beta(-\lambda E(I)) L^{\prime}{ }_{k} \\
& \left.\left.-\beta(-\lambda E(I))^{2} L^{\prime}{ }_{k} E(H)+\beta E\left(L_{k}{ }_{k}\right)(\lambda E(I))^{2}+r E\left(L_{k}{ }^{2}\right)(-\lambda E(1))^{2}\right]\right\}
\end{aligned}
$$

Substituting (33) in (32) we obtain $L_{q}$ in closed form.

Further, all the other queue performance measures can be found using Little's law $W_{q}=\frac{L_{q}}{\lambda^{+}}, \mathrm{W}=\frac{L}{\lambda^{+}}, \mathrm{L}=L_{q}+\rho$

\section{NUMERICAL ILLUSTRATION}

$$
\begin{gathered}
\lambda=4, \beta=2, r=06, m=05, \mu_{\left(e_{1}\right)}=25, \mu_{\left(e_{2}\right)}=35, \mu_{k}=28, \mu_{m}=3, \\
E\left(L_{\left(e_{1}\right)}\right)=\frac{1}{\mu_{\left(e_{1}\right)}}, E\left(L_{\left(e_{2}\right)}\right)=\frac{1}{\mu_{\left(e_{2}\right)}}, L_{k}^{\prime}=\frac{1}{\mu_{k}}, E(H)=\frac{1}{\mu_{m}}, \quad L_{k}(\beta)=\frac{\mu_{k}}{\mu_{k}+\beta}, E\left(L_{k}^{2}\right)=\frac{2}{\mu_{k}^{2}}, \\
E\left(H^{2}\right)=\frac{2}{\mu_{m}^{2}}, L_{k}^{\prime}(\beta)=\frac{\mu_{k}}{\left(\beta+\mu_{k}\right)^{2}}
\end{gathered}
$$

Table 1. Effect of change of ( $\beta=2,25,3,35,4)$.

\begin{tabular}{|c|c|c|c|c|c|}
\hline $\boldsymbol{Q}$ & $\boldsymbol{P}$ & $\boldsymbol{L} \boldsymbol{q}$ & $\boldsymbol{L}$ & $\boldsymbol{W} \boldsymbol{W}$ & $\boldsymbol{W}$ \\
\hline 0.6485 & 0.3515 & 8.5449 & 8.8964 & 2.1362 & 2.2241 \\
\hline 0.6917 & 0.3083 & 12.552 & 12.8602 & 3.138 & 3.2151 \\
\hline 0.7382 & 0.2618 & 16.556 & 16.8173 & 4.1389 & 4.2043 \\
\hline 0.7787 & 0.2213 & 20.983 & 21.2043 & 5.2457 & 5.3011 \\
\hline 0.8190 & 0.1810 & 25.237 & 25.4176 & 6.3092 & 6.3544 \\
\hline
\end{tabular}

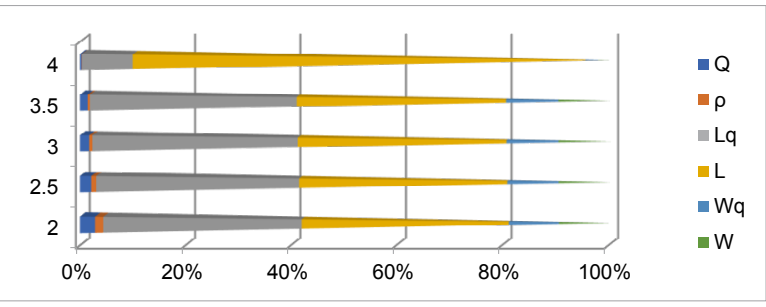

Graphic 3. Effect of change of $\beta$. 
From Table 1 and Figure 3, it is clear that if the probability in service interruption increases it leads to an increase in all the performance measures. Since the service interruption gets increased the idle time gets amplified and utilization factor is decreased.

Table 2. Effect of change of .

\begin{tabular}{|c|c|c|c|c|c|}
\hline $\boldsymbol{Q}$ & $\boldsymbol{P}$ & $\boldsymbol{L q}$ & $\boldsymbol{L}$ & $\boldsymbol{W q}$ & $\boldsymbol{W}$ \\
\hline 0.6485 & 0.3515 & 8.5449 & 8.8964 & 2.1362 & 2.2241 \\
\hline 0.645 & 0.355 & 8.6013 & 8.9563 & 2.1503 & 2.2399 \\
\hline 0.6391 & 0.3609 & 8.6967 & 9.0576 & 2.1742 & 2.2644 \\
\hline 0.6277 & 0.3723 & 8.9197 & 9.2077 & 2.2299 & 2.3019 \\
\hline 0.5977 & 0.4023 & 9.7597 & 10.162 & 2.4399 & 2.5405 \\
\hline
\end{tabular}

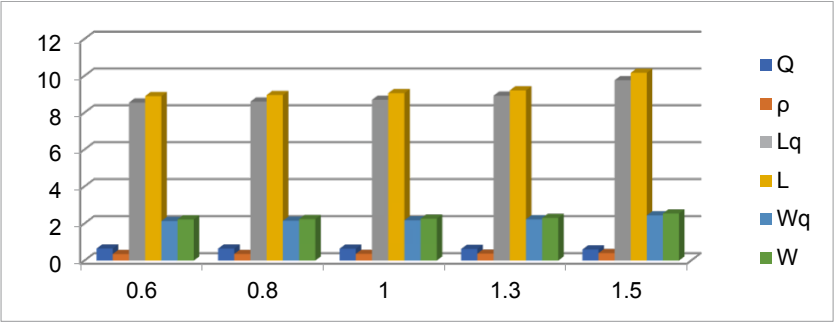

Graphic 4. Effect of change of $r$.

Table 2 indicates that, as the probability of repair rate gets increased, length of the queue is increased. Since the repair rate increased utilization factor gets increased and idle time gets decreased.

\section{CONCLUSIONS}

In this paper we have studied a batch arrival, two phases of essential administration and optional administration, service interruption, feedback service. This paper clearly analyses the steady state results and some queuing performance measures. Further this model can be extended by adding the concept of delay time, reneging, long vacation, short vacation etc. 


\section{REFERENCES}

Blumenthal, D. J., Bowers, J. E., Rau, L., Chou, H. F., Rangarajan, S., Wang, W., \& Poulsen, K. N. (2003). Optical signal processing for optical packet switching networks. IEEE Communications magazine, 41(2), S23-S29. https://doi.org/10.1109/ MCOM.2003.1179494

Boehm, R., Ghing, Y. G., Griffith, G., \& Saal, F. (1986). Standardized Fiber Optic transmission systems-A Synchronous optical network view. IEEE Journal on Selected Areas in Communications, 4(9), 1424-1431. https://doi.org/10.1109/JSAC.1986.1146482

Ghao, H. J., Shtirmer, G., \& Smoot, L. S. (1989). H-Bus: an experimental ATM-based optical premises network. Fournal of Light wave Technology, 7(11), 1859-1867. https:// doi.org/10.1109/50.45908

Cosares, S., Deutsch, D. N., Saniee, I., \& Wasem, O. J. (1995). SONET Toolkit: A Decision support system for Designing Robust and Cost-Effective Fiber-Optic Networks. Informs fournal on applied analytics, 25(1), 1-142. https://doi.org/10.1287/ inte.25.1.20

Hac, A., \& Mutlu, H. B. (1989). Synchronous optical network and broadband ISDN protocols. IEEE Computer, 22(11), 26-34. http://doi.org/10.1109/2.43524

Jue,J. P ., Yang, W. H., Kim, Y. G., \& Zhang, Q. (2009). Optical packet and burst switched networks: a review. IET communications, 3(3), 334-352. http://doi.org/10.1049/ietcom:20070606

Kang, G. S., Park, B. S., Shin, J. D., \& Jeong, J. M. (1995). A broadband ring network: Multichannel optical slotted ring. Computer Networks and ISDN Systems, 27(9), 13871398. https://doi.org/10.1016/0169-7552(94)00055-X

Lee, Y., Sherali, H. D., Han, J., \& Kim, S. (2000). A branch-and-cut algorithm for solving an intraring synchronous optical network design problem. Networks an International fournal, 35(3), 223-232. https://doi.org/10.1002/(SICI)10970037(200005)35:33.0.CO;2-J 
Maragathasundari, S., \& Balamurugan, B. (2015). A study on the performance analysis of a batch arrival queue with two stages of service Bernoulli schedule vacation extended vacation and service interruption. International journal of computer applications, 124(12), 33-37. https://www.researchgate.net/publication/295513726_A_Study_ on_the_Performance_Analysis_of_a_Batch_Arrival_Queue_with_Two_Stages_ of_Service_Bernoulli_Schedule_Vacation_Extended_Vacation_and_Service_ Interruption

Maragathasundari, S., \& Dhanalakshmi, K. S. (2018). Mobile adhoc networks problem-A queuing approach. International journal of communication networks and distributed system, 21(4). https://www.researchgate.net/profile/Dhanalakshmi_ Sadhasivan/publication/325987340_Mobile_Adhoc_Networks_MANETs_ Problem-_A_Queueing_approach/links/5c6270c792851c48a9cd5656/MobileAdhoc-Networks-MANETs-Problem-A-Queueing-approach.pdf

Maragathasundari, S., \& Sowmiah, S. (2016). A study on the analysis of performance measure of bulk input queue with $\mathrm{N}$ type of additional optional service, service interruption and deterministic vacation. Imperial fournal of Interdisciplinary Research, 2(8), 1436-1444.

Maragathasundari, S., \& Srinivasan, S. (2012). Analysis of M/G/1 feedback queue with three stages and multiple server vacation. Applied mathematical sciences, 6(125), 6221-6240. http://m-hikari.com/ams/ams-2012/ams-125-128-2012/ maragathaAMS 125-128-2012.pdf

Maragathasundari, S., \& Srinivasan, S. (2015). A Non-Markovian Multistage Batch arrival queue with break down and reneging. Mathematical problems in engineering, Article ID 519579. http://dx.doi.org/10.1155/2014/519579

Way, W. I., Smith, D. A., Johnson, J. J., \& Izadpanah, H. (1992). A self-routing WDM high-capacity SONET ring network. IEEE Photonics Technology Letters, 4(4), 402-405. https://doi.org/10.1109/68.127228 